\title{
A Performance-Driven Incentive-Based Approach for Successful Service Delivery
}

\author{
Olivia Fachrunnisa ${ }^{1,2}$ \\ ${ }^{1}$ DEBI Institute, Curtin University, Perth, Australia \\ ${ }^{2}$ Dept. of Management, Faculty of Economics, UNISSULA, Semarang, Indonesia \\ E-mail: O.Fachrunnisa@curtin.edu.au.edu.u
}

\begin{abstract}
This paper proposes an appropriate incentive scheme design for service delivery systems to ensure service deliverability. We use proactive continuous performance monitoring to provide qualitative observations and data on how well services are being provided and whether desired service outcomes are being achieved as a result of interaction between two parties (service provider and service requester). The paper provides a general framework for analyzing the effectiveness of incentive and proactive continuous monitoring in ensuring successful service delivery. The objective of the incentive is to motivate the service provider to deliver service as agreed to by both parties. We show the robustness of the framework by running several simulations under various conditions. It is shown that proactive continuous monitoring and incentive are essential for increasing performance which will result in successful service delivery.
\end{abstract}

Keywords: incentive, service delivery, digital ecosystems

\section{INTRODUCTION}

The new paradigm of Digital Ecosystems is built not only on transactions but on establishing, sustaining and improving relationships with existing or potential stakeholders. Hence, trust is always seen as a value which strengthens the business relationship [1, 2]. A key research issue relating to the formation of a successful business relationship is service guarantee and service deliverability. However, the existing literature does not focus on this. By 'service deliverability', we mean a guarantee of continued delivery of service as mutually agreed to by both of the parties involved. A business relationship depends on the strength of the trust and commitment both interacting parties to deliver service according to the terms of a mutual agreement. In this paper, we consider 'trust level' to be a representation of quality of service delivered. Quality of Service (QoS) is defined as 'the fulfillment of the service agreement or mutually agreed service' [3]. Trust is the degree of belief or faith that an interacting party has in another party [3] that forms the basis of QoS and the subsequent establishment of a business relationship [4]. Hence, trust level is the extent to which the trusted agent is capable of delivering service as mutually agreed to by both parties.

In this paper, we present an incentive-based mechanism to improve the performance of a trusted agent in delivering service to the trusting agent. The incentive is an increase in the performance level of the trusted agent in delivering agreed service; hence, we term it 'performance-driven, incentivebased'. Reward and incentive are widely acknowledged as motivators which can encourage agents to improve their performance. Badenfelt [5] stated that trusting agents may use rewards to change the trust levels in organizations. Although several researches have shown that the use of incentives can successfully increase the performance of agents in a nonvirtual environment, Ferrin and Dirks [6] suggested that the effects of rewards in trust building in the context of virtual environment should be examined.

In order to support an incentive mechanism, we propose a proactive continuous performance monitoring mechanism for interactions between trusting agents and trusted agents. It is designed to monitor the service delivery at every checkpoint. Checkpoints are intermediate milestones that are created in order to check service delivery during the time space of a transaction. With our mechanism, a service task is decomposed into several intermediate deliveries to ensure the continuity of service delivery until the final desired outcome has been achieved. The main purposes of this checkpoint mechanism are to identify early disagreement (or noncompliance) between actual performance and mutually agreed performance and to resolve any issue(s).

This continuous monitoring enables performance variances to be timely identified. Consequently, both parties will be able to quickly close the performance gap before it inflates into an unmanageable and undelivered service by end of the transaction. The incentive would be used to encourage trusted agents to always comply at each checkpoint. It will guarantee successful delivery of that service. If an agent has not fully delivered service at a particular checkpoint, the remaining undelivered service needs to be delivered at the next checkpoint. If at this checkpoint, the agent increases his compliance performance compared to the previous checkpoint, then this agent will receive an incentive. As a result of receiving an incentive, their performance level at the next checkpoint will increase. We present details of this mechanism in the next section.

The rest of the paper is organized as follows. Section 2 presents a model overview. Section 3 presents the experiment results. In Section 4, we validate the framework by means of a series of experiments. We briefly review the current researches in the field of incentives and rewards for increasing performance in Section 5. Conclusions are drawn and future works are outlined in the final section. 


\section{BACKGROUND AND RELATED WORK}

Several existing works suggest that the level of trust in such service relationships should always be monitored to ensure the success of service delivery. Therefore, although trust has been established between the trusting agent and trusted agent in a transaction relationship, monitoring still plays an important role in ensuring that the partnership relationship continues to operate as intended [7, 8]. Henceforth, even in situations where there is a sufficient level of trust between trusting agents, this trust needs to be underpinned by robust monitoring and, where necessary, reward and sanctioning processes could be utilized $[6,9]$.

Such continuous performance monitoring activities enable performance discrepancies to be identified early and be resolved. Kumaran et al. [10] proposed a MDBT (Model Driven Business Transformation) for transaction monitoring between service provider, outsourcer and service requester. It provides a service delivery performance management platform which includes notification of any violation of SLAs. The performance is monitored by comparing actual performances against Key Performance Indicators (KPIs). The platform aims to model performance metrics for service delivery and describe how the monitoring and management of KPIs are supported as an integral part of SDM (Service Delivery Management) platform. However, this platform does not provide any mechanism, using reward and incentive, to motivate the service provider to fully comply with SLAs.

Reward and incentive have been acknowledged as factors which contribute to the success of interactions or to increase the involvement of interacting parties [11]. Long and Sitkin [9] examine the various ways to balance interpersonal trustbuilding and control-based efforts in order to maintain trustbased relationships. Written contracts, surveillance and monetary incentives are examples of formal mechanisms for controlling the delivery of service from trusted agent to trusting agent. They maintain that trusting agents can use incentive mechanisms to direct trusted agents toward the efficient and effective completion of tasks. However, the incentive is given after the completion of tasks at the end of the interaction.

Ferrin and Dirks [6] carried out laboratory experiments to examine whether rewards have a direct effect on trust or whether they represent a catalyst that may set in motion other processes that influence trust. Their research concludes that rewards have a strong impact on the formation of trusting beliefs. However, the incentive is given after the trusted agent had completely delivered the service. They do not propose any mechanism that provides an incentive at intermediate intervals during the delivery of service.

Unlike the existing works in the literature regarding incentives for task completion, in this paper we combine a mechanism of proactive continuous monitoring and performance-driven incentive specifically to support successful service delivery. The service delivery is monitored at intermediate checkpoints and incentives are given at intermediate checkpoints rather than at the end of the interaction. We do not intend to provide an incentive after task completion; instead, it is given during the course of the transaction to minimize the discrepancy between actual performances and mutually agreed to performance.

\section{MODEL OVERVIEW}

The objective of our system is to motivate trusted agents to maintain their performance in delivering service to the trusting agent. We propose to include the following properties in the design of our incentive mechanism:

1. Mutually Agreed Performance. For each transaction, both parties have agreed on mutually agreed performance. This agreement clearly states each service criterion and its associated level of importance.

2. Time Space. Before each transaction, the trusting agent and trusted agent determine the entire duration of time over which the complete delivery of agreed service will occur. We term this the 'time space'.

3. Check point. In order to ensure the continuity of service delivery, the trusting agent divides the time space into a certain number of checkpoints. At each checkpoint, the agreed performance of service delivery is expressed. This checkpoint system is intended to facilitate continuous performance monitoring during the interaction. With the designed checkpoints, the trusting agent can observe or track changes in the performance of the trusted agent over a time space.

4. Intermediate Performance. The performance of the trusted agent in terms of delivering the service is monitored at each checkpoint. It is reflected in the correlation between actual service and mutually agreed upon service at each checkpoint. Hence, at each checkpoint, the trusting agent will allocate a value of intermediate performance. We measure the performance of trusted agent by correlating actual performance with mutually agreed performance. The intermediate performance is calculated using the following formula:

$$
P_{\mathrm{int}} \mathrm{CPn}=6 *\left(\frac{\sum_{C=1}^{N} \text { In Actual Perf }_{\text {criterionc }} * \text { Clear }_{\text {criterionc }} * \operatorname{Imp}_{\text {criterionc }}}{\sum_{C=1}^{N} \operatorname{In} M A P_{\text {criterionc }} * \text { Clear }_{\text {criterionc }} * \operatorname{Imp}_{\text {criterionc }}}\right)
$$

Where,

$P_{\text {int }} C P n$ denotes intermediate performance at
checkpoint $\mathrm{n}$

6 represents the highest level of performance

In Actual Perf $f_{\text {criterionc }}$ denotes the actual / delivered criterion representing the fulfillment of each criterion of the trusted agent at an intermediate checkpoint. 
InMAP $P_{\text {criterionc }}$ denotes mutually agreed performance of the trusted agent according to the given criterion at the intermediate checkpoint.

Clear $_{\text {criterionc }}$ represents the clarity of each criterion in the service agreement (contract) and whether it is understood in the same way by both parties. For a given criterion, Clear $_{\text {criterionc }}$ can have two levels (a) ' 0 ' - This criterion or its output or both have not been mutually agreed to by both parties and (b) ' 1 ' - This criterion along with its output has been mutually agreed to by both parties.

Imp $_{\text {criterionc }}$ denotes the importance of each criterion that affects the determination of trustworthiness. For a given criterion, $\operatorname{Imp}_{\text {criterionc }}$ can have three levels: (a) '0' - not important (b) ' 1 ' - important, and (c) '2' very important.

5. Incentive $C P$. The incentive at each checkpoint will be issued if the performance of a trusted agent at the current checkpoint is higher than for the previous checkpoint. Hence, if Pint $_{C P n}>$ Pint $_{C P n-1}$, the trusting agent will give an incentive to the trusted agent, while Pint $_{C P n}$ is intermediate performance at current checkpoint and Pint ${ }_{C P n-1}$ is the intermediate performance at the previous checkpoint. We term the checkpoint which receives the incentive as the 'incentive $C P$ '. During one transaction, there would be several checkpoints. It is important to note that the incentive is given only if the agent has shown an increase in performance.

6. Incentive value. The trusting agent will give an incentive value as a kind of 'trust value'. In this paper, the highest trust value as an incentive during a transaction is ' 1 '. Hence, the value of incentive for each 'incentive CP' is calculated as follows:

$$
\text { Incentive } \mathrm{CP}=\frac{1}{\text { total number of } \mathrm{CP}}
$$

7. Increasing performance as a response of incentive. Once an agent has received an incentive at a certain checkpoint, as a response to this incentive, he increases his performance level for the next checkpoint. The level of increasing performance is calculated as follows:

IPL = CLCPn * initial performance level * brake option

Where IPL denotes as increasing performance level, $\mathrm{CL}_{\mathrm{CPn}}$ denotes the compliance level at checkpoint $\mathrm{n}$. This $\mathrm{CL}_{\mathrm{CPn}}$ is chosen randomly by the system at each initial checkpoint. Initial performance is randomly assigned early in the simulation. Brake option is a value of $0-0.9$, which represents the degree of performance increasing after receiving an incentive.

8. Final Performance. At the end of the interaction or time space, the final performance as a representation of successful service delivery is calculated by aggregating the intermediate performance during the transaction. It can be calculated by using the following equation:

$$
\mathrm{P}_{\text {final }}=\left(\frac{\sum_{\mathrm{c}=1}^{\mathrm{N}} P_{\mathrm{int}}}{\mathrm{n}}\right)
$$

While $P_{\text {int }}$ is performance at intermediate point and $n$ denotes the total number of check points in an interaction. $P_{\text {int }}$ value can be calculated by using (2) above.

Let us consider the following case study for elucidation purposes. Agent 'A' is a trusting agent who requests that agent ' $B$ ' delivers 1000 service units for the duration of one month time space. Agent ' $A$ ' then divides the time space into 4 checkpoints. At each checkpoint, it is agreed that agent ' $\mathrm{B}$ ' will deliver 250 units. As explained previously, the checkpoint is designed to monitor the progress of performance continuously rather than at the end of the interaction.

Let us consider that at the first checkpoint, agent ' $\mathrm{B}$ ' delivers only 200 units which is below the intermediate mutually agreed performance. At this first checkpoint, by using (1), the intermediate performance for this checkpoint 1 is 4.8. In our model, we propose a mechanism by which the performance gap at the previous checkpoint is handed over to the next checkpoint. Hence, in this case, the agreed performance for the second checkpoint is 300 units instead of 250 units. If at this checkpoint, the ratio of actual performance against the mutually agreed performance is greater than checkpoint 1 , this checkpoint is characterized as an incentive checkpoint. For example, if the agent delivers 290 units, using (1) the $P_{\text {int }} \mathrm{CP}_{2}$ is 5.8. As $P_{\text {int }} \mathrm{CP}_{2}$ is greater than $P_{\text {int }} \mathrm{CP}_{1}$, this checkpoint 2 is marked as incentive CP.

As a result of receiving the incentive, this agent will increase his compliance level at checkpoint 3 . The increasing performance level is calculated by using (3). This mechanism continues until the end of the transaction time space. Finally, at the end of the transaction, we measure the final performance as the aggregate value of intermediate performances. If the final performance is greater than the initial performance, we may conclude that incentive has facilitated the successful delivery of service by increasing the agent's performance level.

The initial performance and final performance are compared in order to show that the trusted agent has been increasing his performance level in response to receiving an incentive. This will lead to the completion of service delivery from trusted agent to trusting agent. 


\section{IMPLEMENTATION AND RESULTS}

In order to determine the effectiveness of our mechanism, we engineered a multi-agent system using the JADE Multi Agent-Based Framework which has an interface enabling the user to specify the parameters prior the execution. The user can specify the total number of interactions (or transactions) that need to be simulated. Additionally, the user specifies the total number of agents in the multi-agent system that needs to be classified into six classes, with each class corresponding to one particular trustworthiness value and associated compliance level.

During the bootstrapping phase, the agents are created and each of them is assigned a given behavior, to represent the degree of their performance level. Hence, each agent would have an initial performance level. Each performance level corresponds to one trustworthiness value. For example, the behavior belonging to agents with trustworthiness value ' 1 ' $\left(\mathrm{TV}_{1}\right)$ would contain a behavior that would specify the action or the way that agents carry out a transaction. Hence, we propose that the performance level of an agent is the degree to which an agent complies with mutually agreed performance.

Since there are six different trustworthiness values, there are six different performance behaviors. In this simulation, we propose a correctness of agent's compliance level using Fuzzy Trapezoidal Performance Level. In this approach, the behavior of each agent corresponding to a trustworthiness value is represented using a membership function as shown in Table 1. The degree of compliance behavior of an agent in each trust value is determined with the help of a statistical graph which corresponds proportionally to the measurement of 100 scale intervals. The number ' $0 \%$ ' denotes the lowest level of compliance, meaning that the agent is totally non-compliant, and ' $100 \%$ ' denotes perfect performance or full compliance. A fuzzy trust grade set is defined as the fuzzy measurement result, which is denoted by Trust Value $(\mathrm{TV})=[1,2,3,4,5$, 6]. These six grades $\mathrm{TV}_{1}, \mathrm{TV}_{2}, \mathrm{TV}_{3}, \mathrm{TV}_{4}, \mathrm{TV}_{5}$, and $\mathrm{TV}_{6}$ denote the gradational measurement results ranging from the fully compliant to fully non-compliant as depicted in Fig. 1 below.

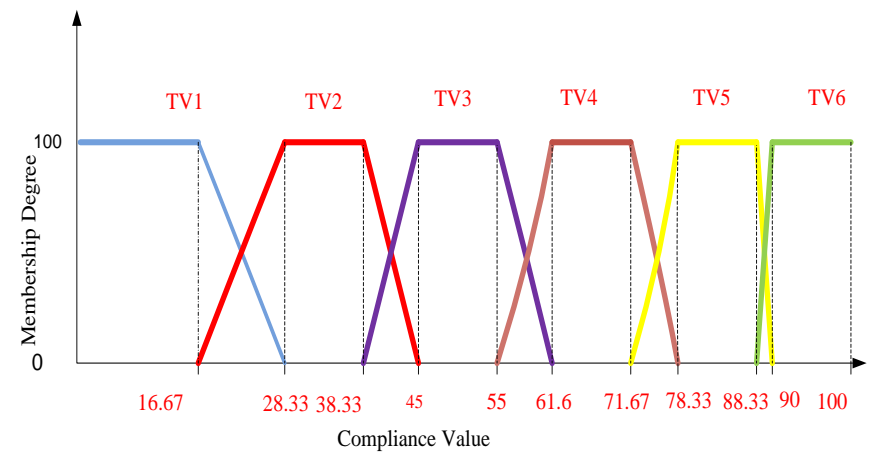

Fig 1. Trapezoidal Membership Degree and Compliance Value.

The graph above shows the degree of membership and value of compliance for every agent in each trust value. It can be seen that the nature of membership functions is primarily trapezoidal; hence, we term this set of compliance 'fuzzy trapezoidal compliance'. An agent with a certain level of compliance will have a certain trust value. Membership degree (y) which ranges from 0 to 1 is a mapping called the membership function of the fuzzy set TV, and level of compliance $(x)$ indicates the degree of belongingness or membership value of ' $x$ ' in any Trust Value. Therefore, a certain compliance level acquires two trustworthiness (membership) characteristics.

During simulation, the user randomly selects an agent with the probability of having a compliance value ranging from $0 \%$ to $100 \%$. There are six grades of $\mathrm{TV}_{1}, \mathrm{TV}_{2}, \mathrm{TV}_{3}, \mathrm{TV}_{4}, \mathrm{TV}_{5}$, and $\mathrm{TV}_{6}$. Each compliance value will correspond to a certain degree of trust value membership. For example, if the user chooses an agent with level of compliance $28 \%$, his behavior will express some degree similarity of $T_{1}$ and $T_{2}$ characteristic as this point has a certain degree of membership in $\mathrm{TV}_{1}$ and $\mathrm{TV}_{2}$. However, the total degree of membership is always 1 .

The equations representing fuzzy trapezoidal compliance corresponding to $\mathrm{TV}_{1}, \mathrm{TV}_{2}, \mathrm{TV}_{3}, \mathrm{TV}_{4}, \mathrm{TV}_{5}$, and $\mathrm{TV}_{6}$ are shown below:

TABLE 1

TRAPEZOIDAL COMPLIANCE LEVEL FUZZY RULE

\begin{tabular}{|c|c|c|}
\hline TV & Membership Degree & $x$ value \\
\hline \multirow{4}{*}{$\mathrm{TV}_{1}$} & 0 & $x \geq 28.33$ \\
\hline & $28.33-x$ & \multirow{2}{*}{$16.67<x<28.33$} \\
\hline & $\overline{28.33-16.67}$ & \\
\hline & 1 & $0 \leq x \leq 16.67$ \\
\hline \multirow{4}{*}{$\mathrm{TV}_{2}$} & 0 & $x<16.67$ \\
\hline & $\frac{16.67-x}{28.33-16.67}$ & $16.67<x<28.33$ \\
\hline & 1 & $28.33 \leq x \leq 38.33$ \\
\hline & $\frac{45-x}{45-38.33}$ & $38.33<x<45$ \\
\hline \multirow{4}{*}{$\mathrm{TV}_{3}$} & 0 & $0 \leq x \leq 38.33$ \\
\hline & $\frac{x-38.33}{45-38.33}$ & $38.33<x<45$ \\
\hline & 1 & $45 \leq x \leq 55$ \\
\hline & $\frac{61.67-x}{61.67-55}$ & $55<x<61.67$ \\
\hline \multirow{4}{*}{$\mathrm{TV}_{4}$} & 0 & $0 \leq x \leq 55$ \\
\hline & $\frac{x-55}{61.67-55}$ & $55<x<61.67$ \\
\hline & 1 & $61.6 \leq x \leq 71.67$ \\
\hline & $\frac{78.33-x}{78.33-71.67}$ & $71.68<x<78.33$ \\
\hline \multirow{4}{*}{$\mathrm{TV}_{5}$} & 0 & $0 \leq x \leq 71.67$ \\
\hline & $\frac{x-71.67}{78.33-71.67}$ & $71.67<x<78.33$ \\
\hline & 1 & $78.33 \leq x \leq 83.33$ \\
\hline & $\frac{90-x}{90-88.33}$ & $88.33<x<90$ \\
\hline \multirow{3}{*}{$\mathrm{TV}_{6}$} & 0 & $0 \leq x \leq 88.33$ \\
\hline & $\frac{x-83.33}{90-88.33}$ & $88.33<x<90$ \\
\hline & 1 & $90<x \leq 100$ \\
\hline
\end{tabular}


In this approach, consider for example that we have an agent with a compliance level of $60 \%$. This agent will behave according to some degree of $\mathrm{TV}_{3}$ and to some extent of $\mathrm{TV}_{4}$. Therefore, the calculation of compliance behavior is as follows:

Compliance Behavior $=\left(\right.$ membership degree $* \mathrm{TV}_{3}$ char $)+$ (membership degree* TV 4 char)

Membership degree is the level of membership based on calculation using the fuzzy rule above, while $T_{\mathrm{n}}$ char is a characteristic of trust value as derived from the crisp compliance level. Based on the above rule, the compliance level of this agent is $62.5 \%$ as a result of the following calculation:

TABLE 2

\section{COMPLIANCE BEHAVIOR CALCULATION}

\begin{tabular}{|c|c|c|l|}
\hline $\mathrm{TV}$ & $\mathrm{x}$ value & Membership Degree & $\begin{array}{l}\text { Compliance } \\
\text { Behaviour }\end{array}$ \\
\hline $\mathrm{TV}_{3}$ & $\frac{61.67-x}{61.67-55}$ & $\frac{61.67-60}{61.67-55}$ & $0.25 * 50=12.5$ \\
\hline $\mathrm{TV}_{4}$ & $\frac{\boldsymbol{x}-55}{\mathbf{6 1 . 6 7 - 5 5}}$ & $\frac{60-55}{\mathbf{6 1 . 6 7 - 5 5}}$ & $\begin{array}{c}0.75 * 66.67= \\
50.0025\end{array}$ \\
\hline Total & & $0.75+0.25=1$ & $\begin{array}{c}12.5+50.0025 \\
=62.5025\end{array}$ \\
\hline
\end{tabular}

Moreover, the user can also specify the bonus bias which ranges from 0.1 to 0.9 . This bonus bias is a parameter to determine the increasing behavior of an agent after an incentive has been given.

In order to show the effectiveness of the incentive mechanism in supporting successful service delivery, we compared the simulation results from transactions with incentive and without incentive. In transactions without an incentive mechanism, if an agent performs better than at the previous checkpoint, there is no incentive and no increasing performance for the next checkpoint as well.

Figure 2 below shows the comparison result from simulation of interactions with incentive and without incentive from 1000 agents who carried out 1000 transactions; bonus bias in the incentive mechanism benchmark is 0.9 .

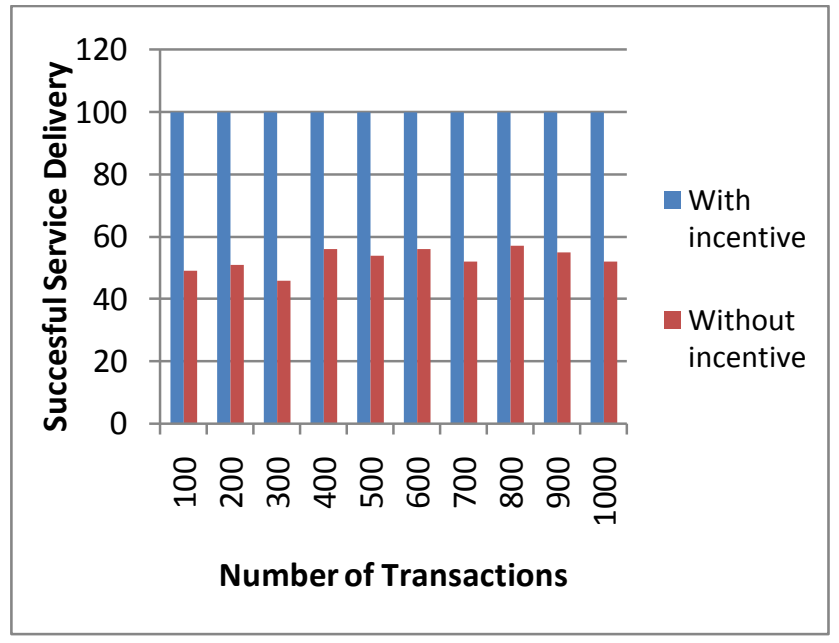

Fig. 2. 1000 agents carried out 1000 transactions (bonus bias 0.9)

As we can see from figure 2 above, the successful service delivery resulting from a transaction with incentive is higher than for a transaction without incentive. We measure the successful delivery as a comparison between initial performance level and final performance level. If the final performance level is greater or equal than the initial performance level, then it indicates that an agent in that particular interaction has increased his compliance level. It does not mean that the service has been delivered at a full compliance level (100\%); rather, it depends on the initial compliance level as a representation of the trustworthiness value of agent.

As observed in interactions with incentive, the successful service delivery remains $100 \%$ throughout the interaction. It means that all agents have shown an increase in performance as a result of the incentive. In contrast, for interactions without incentive, the successful service delivery is less than $60 \%$. This means that during transactions, only $60 \%$ of agents have shown improved behavior.

Figure 3 below illustrates the results of an experiment using transactions with 3000 agents who carried out 2000 transactions and the bonus bias is 0.9 .

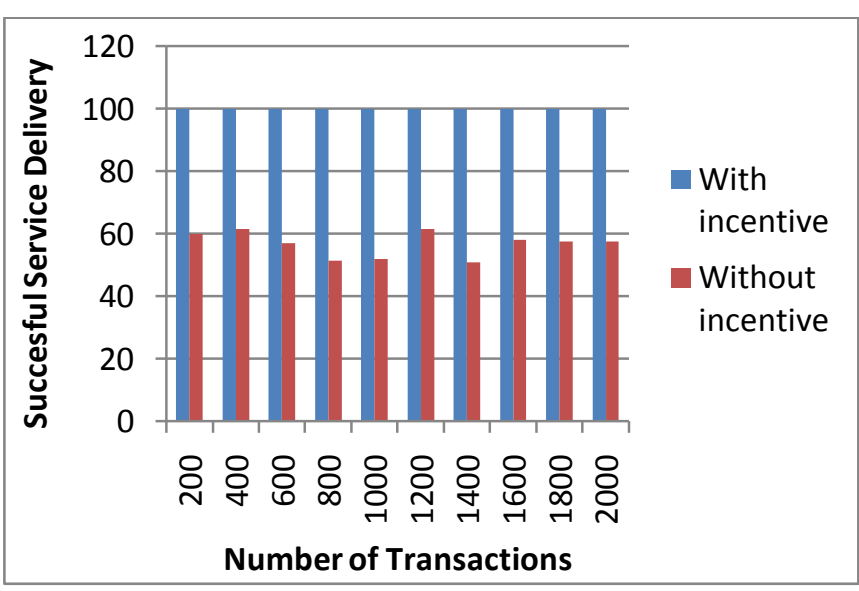

Fig. 3. 3000 agents carried out 2000 transactions (bonus bias 0.9). 


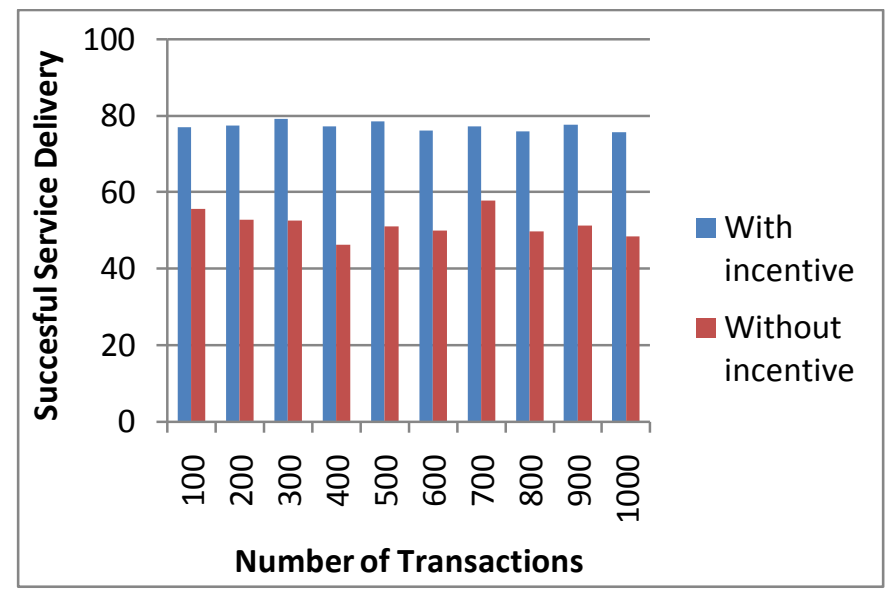

Fig. 4. 2500 agents carried out 5000 transactions (bonus bias 0.5)

It is also observed from Figures 3 and 4 that with the larger number of agents and interactions, there are significant differences between two transaction scenarios. Interactions with an incentive mechanism have a higher rate of success than do those interactions without an incentive mechanism.

It can be inferred from the simulation results, that incentive is effective as a means of increasing the performance of trusted agents to deliver services to the trusting agents at each checkpoint. Moreover, the proactive continuous monitoring with checkpoints is designed to support the continuity of service delivery. With the proactive continuous monitoring, the trusting agent will be updated about the performance of the trusted agent and this will help to close the performance gap in real time. Hence, it guarantees service deliverability at the end of transactions.

\section{CONCLUSION AND FUTURE WORK}

In this paper, we present a mechanism for proactive continuous monitoring and performance-driven incentive in business interactions between service providers and service requesters. These two mechanisms are intended to support the successful delivery of service. At each transaction, the service task is decomposed into several intermediate deliveries as agreed to by both parties. Hence, the service delivery is monitored at intermediate checkpoints and incentive is given at intermediate checkpoints if the trusted agent has shown an increase in performance.

Unlike existing works, incentive is given at transaction milestones as a part of continuous performance monitoring, rather than at the end of transaction. If an agent performs consistently according to the mutually agreed performance at each checkpoint, then this agent receives an incentive. As a result of this, the agent will increase his performance or compliance level. Therefore, any performance gap is identified and resolved in real time. It will guarantee the successful of delivery of service by the end of the transaction.
In our future works, we intend to introduce another scheme of incentive according to the degree of compliance level. We also plan to design a penalty system for unsuccessful service delivery.

\section{REFERENCES}

1. Debenham, J. Modelling trust, honour and reliability in business relationships. in 3rd IEEE International Conference on Digital Ecosystems and Technologies, 2009. DEST '09. . 2009.

2. Giannoutakis, K.N. and M. Petrou. The effect of trust in product chains in a Digital Business Ecosystem. in Inaugural IEEE-IES Digital EcoSystems and Technologies Conference, 2007. DEST '07. . 2007.

3. Chang, E., T.S. Dillon, and F.K. Hussain, Trust and reputation for service-oriented environments : technologies for building business intelligence and consumer confidence. 2006, Chichester, England: John Wiley \& Sons Inc. xix, 349 p.

4. Ruppel, C., L. Underwood-Queen, and S.J. Harrington, e-Commerce: The Roles of Trust, Security, and Type of e-Commerce Involvement. eService Journal, 2003. 2(2): p. 25-45.

5. Badenfelt, U., I trust you, I trust you not: a longitudinal study of control mechanisms in incentive contracts. Construction Management and Economics, 2010. 28(March): p. 301-310.

6. Ferrin, D.L. and K.T. Dicks, The use of rewards to increase and decrease trust: mediating processes and differential effects. Organization Science, 2003. 14(1): p. 18-31.

7. Sullivan, R., Building Trust through Governance: Lessons from Tri-sector Partnerships in the Extractive Industries. Journal of Corporate Citizenship, 2007. Spring: p. 55 - 75.

8. Skopik, F., D. Schall, and S. Dustdar, Modeling and mining of dynamic trust in complex service-oriented systems. Information Systems, 2010. 35(7): p. 735757.

9. Long, C.P. and S.B. Sitkin, eds. Trust in the balance: how managers integrate trust-building and task control. Handbook of Trust Research, ed. R. Bachmann and A. Zaheer. 2006, Edward Elgar: Massachussetts.

10. Kumaran, S., et al., Using a model-driven transformational approach and service-oriented architecture for service delivery management. IBM Systems Journal, 2007. 46(3): p. 513-529.

11. Ranganathan, K., et al., To Share or Not to Share: An Analysis of Incentives to Contribute in Collaborative File Sharing Environments, in Workshop on Economics of Peer-to-Peer Systems. 2003. 